\title{
Energy and Density Aware Clustering (EDAC) to Improve Lifetime and Connectivity in Multi Level Heterogeneous Wireless Sensor Network
}

\author{
Ajit Singh Negi \\ M.Tech Scholar \\ Dept. of CSE \\ Graphic Era University \\ Uttarakhand, Dehradun, India
}

\author{
Neha Garg \\ Assistant Professor \\ Dept. of IT \\ Graphic Era University \\ Uttarakhand, Dehradun, India
}

\author{
Devesh Pratap Singh \\ Assistant Professor \\ Dept. of CSE \\ Graphic Era University \\ Uttarakhand, Dehradun , India
}

\begin{abstract}
Network lifetime and connectivity are the key parameters to guarantee quality of service (QoS). As a result it becomes our top most priority to preserve the energy of sensor nodes which have limited initial energy at the time of deployment and it is impossible to recharge the battery due to out of reach deployment. Heterogeneous wireless sensor network consists of more than one type of sensor nodes which differs from one another in terms of initial energy, computing power, performance, etc. In order to reduce unwanted energy dissipation and congestion during long distance data transmission, fixed number of relay nodes are selected and placed as a router to maintain connectivity. Relay nodes receive aggregated data from cluster head and send it back to base station. In this paper, we have proposed an (EDAC) Energy and Density Aware clustering scheme to optimize the selection and placement of cluster heads $(\mathrm{CH})$ and relay Nodes (RN) to enhance the connectivity and network lifetime of network. The proposed centralized protocol enables the base station to select the cluster head and relay node on the basis of residual energy, distance from base station and relay node, node density, overlapping ratio of nodes, etc. The results of simulation using Matlab proves that our proposed algorithm, EDAC increases the overall connectivity , 100\% coverage ratio by $80.21 \%$ and network lifetime by $131.61 \%$.
\end{abstract}

\section{General Terms}

Wireless Sensor Networks, Clustering

\section{Keywords}

Network Lifetime, Connectivity, Heterogeneous Wireless Sensor Network, Energy Efficiency, Cluster Head, Relay Node, Routing, Static Clustering.

\section{INTRODUCTION}

Wireless sensor network (WSN) is a collection of tiny sensors deployed to monitor [1] [22] and gather information from area of interest. WSN can be either Homogeneous where all nodes have same energy level, computing power, processing power, etc. or it can be Heterogeneous. Heterogeneous wireless sensor network as shown in Figure 1.1 [4] contains two, three or multiple type of nodes which differ with each other with respect to their energy level, computing power, processing power, memory, etc. and are termed as two, three or multilevel Heterogeneous WSN [16] respectively based on the different types of nodes used like normal ,advance, super nodes, etc. Clustering [2] based routing techniques is one of the most important source of energy conservation for sensor networks where resources are limited. Sensors have limitation with respect to their initial energy level, processing power, sensing ability and memory. Therefore, a network of these sensors leads to failure of network resulting in loss of connectivity, lifetime and loss of valuable data during heavy traffic. This problem can be avoided by using heterogeneous nodes having variation in initial energy, processing power, etc. and implementing clustering along with the placement of relay nodes resulting in multi hop architecture. An inefficient use of the available energy leads to poor performance and short life cycle of the network. Thus it can be concluded that by using proper multi hop communication between sensor nodes and base station (BS) via relay nodes can result in energy conservation of nodes which are limited in energy resources. Selection and placement of relay nodes and cluster head are the key challenge for designing energy and density aware networks. Sensor nodes deployed in monitoring area need to be divided further into individual subsets in order to send traffic to a relay nodes via cluster head.

In this work, EDAC, an Energy and Density Aware Clustering protocol to improve the connectivity and network lifetime of multi level heterogeneous WSN is proposed. It is a modification of existing routing protocol, CCPR [13] and is based on ECHR [12] for deriving cost metrics used in EDAC and is purely a centralized algorithm. Implementing [19] static or fixed size clusters along with dynamic cluster head election in EDAC is ideal for application like air pollution monitoring [18], radioactive emission detection, etc. where monitoring area is divided into fixed size sub area's resulting in better management of data. Proposed EDAC scheme try to preserve energy wasted in long distance data transmission and improves low connectivity faced by nodes while sending data to BS by optimal selection and placement of fixed number of relay nodes and cluster head unlike other routing protocol where number of $\mathrm{CH}$ generated are higher and unbalanced resulting in extra overhead computation. Base station select the cluster head and relay node on the basis of residual energy, distance from base station and relay node, node density, overlapping of nodes, etc. to make network energy and density aware resulting in reduction of communication cost to prolong lifetime and maintain optimal connectivity.

This rest of the paper is organized into following subsection: section II contains the related research works done, section III explains the network, coverage and radio energy dissipation model, section IV presents the proposed EDAC algorithm , section $\mathrm{V}$ shows the simulation, section VI highlights conclusions \& future scope and section VII shows references. 


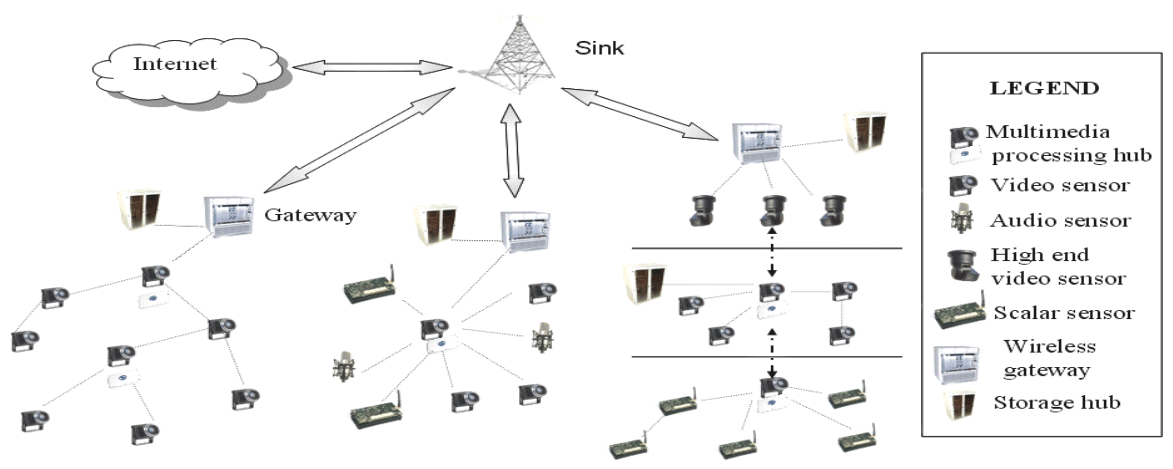

Figure 1.1: Example of Multi Level Heterogeneous Wireless Sensor Network deployed for Wild Life Monitoring [4]

\section{RELATED WORK}

Clustering algorithm applied in homogeneous network are known as homogeneous clustering schemes and those which are applied in heterogeneous network are termed as heterogeneous clustering schemes LEACH [6], Q-LEACH [17], etc. where all sensor nodes have same initial energy are example of homogeneous clustering scheme.SEP [7], DB-SEP [8], DEEC [9], etc. are example of heterogeneous clustering scheme. At present majority of application which are performing multiple and time critical sensing operation are based on heterogeneous wireless sensor network.

One of the most successful distributed clustering routing protocol in WSN literature is Low Energy Adaptive Clustering Hierarchy, LEACH [6]. During the initialization phase, LEACH execute cluster head selection. To achieve load balancing among all homogeneous nodes, LEACH randomly elects cluster head nodes in each round. The elected cluster head performs fusion and aggregation of data arriving from nodes that belongs to the respective cluster. Later on all the data is sent to base station periodically. LEACH operation consist of two phases namely, the Setup-phase and SteadyState phase. In setup phase, selection and organization of cluster head takes place. During steady state phase, actual data transmission of data from cluster head to base station takes place.

During setup phase node " $n$ " selects a random number, $\mathrm{k}$ between 0 and 1 . Node " $n$ " becomes cluster head for the current rotation round if the number $\mathrm{k}$ is less than the threshold value which is computed as follows:

$$
T(n)= \begin{cases}\frac{P}{1-P *\left(r \bmod \frac{1}{P}\right)} & \text { if } \mathrm{n} \in \mathrm{G} \\ 0 & \text { otherwise }\end{cases}
$$

Here $G$ denotes the super set of sensor nodes that have not been declared as a clustered head in the last $1 / P$ rounds where $\mathrm{P}$ is expected percentage of cluster heads in the network and $\mathrm{r}$ being the current round number. Use of single hop routing along with unbalanced cluster of homogeneous nodes in LEACH during transmission of data from cluster head to base station makes it poor candidate for routing protocol in an application where large network exists due to non uniform energy consumption and absence of connectivity.

SEP [7] introduced by Georgios S. et al. is two level energy based heterogeneous routing protocol which is based on concept of selection of cluster head using weighted election probability for each node according to remaining energy in each node. Here two types of nodes are used in which normal nodes are more in numbers as compared to advance nodes. Advance nodes are having more energy than normal nodes resulting in enhancement of network lifetime and stability period. It works well with small and large scale networks and does not require prior distribution of sensor nodes. Inability to maintain stability and efficient use of remaining energy in multilevel environment is the main limitation of SEP.

DB-SEP [8] , a modified version of SEP , proposed by Said Benkirane et al. is 2 level energy based heterogeneous routing protocol. Objective of this protocol is to increase the lifetime of heterogeneous wireless sensor network by selecting cluster heads on the basis of initial energy, distance between nodes and distance with respect to base station. This scheme is purely probabilistic in nature and more efficient than SEP but not good in terms of energy efficiency and network lifetime.

Li Qing et al. proposed Distributed Energy Efficient clustering, DEEC [9] which provides better network lifetime. In DEEC there is no requirement of having global knowledge of about node energy at every round for cluster head election. Cluster head selection uses probability which is based on ratio of node residual energy and average energy of whole network. In DEEC cluster formation is dynamic which consume extra energy at every new round resulting in decrease in energy.

The performance study of network using simulation , analysis, real test bed measurements etc. for number and placement of heterogeneous sensor nodes in network having different size and density has been evaluated in [10].This study is helpful in deciding where, how many, and what type of heterogeneous nodes to deploy in sensing field. These results are found to be valid for Manhattan grid networks, in which deployment of nodes is done by following grid pattern which is not possible for application where random deployment is done like forest fire detection ,radioactive emission detection, etc.

S. Soro et al. have proposed a set of effective cost metrics [11] that combines residual energy of node along with there contribution in network coverage and connectivity. These metrics are favoring nodes which are deployed in densely populated area as a suitable candidates for cluster head nodes and relay nodes. It helps to prolong network lifetime and connectivity of network.

Joe Air Jiang et al. proposed ECHR [12] , a routing protocol to support energy coverage preservation for sensor nodes in WSN. Main concept behind ECHR is to consider both remaining energy of nodes and coverage redundancy 
associated with its sensing range while selecting root node or cluster head to forward collected data to base station in mutihop and chain like communication. Well spread distribution of homogeneous sensor nodes during deployment is assumed in ECHR but ECHR fails to provide proper coverage and connectivity during random or sparse heterogeneous nodes deployment where it becomes difficult to provide equal energy and well spread distribution resulting in absence of regular links in form of parent node or relay node between sensor nodes. Frequent selection of sensor node having high residual energy and coverage redundancy also leads to failure of connectivity and coverage after certain period of network lifetime due to low residual energy is left in sensor node's.

CCPR [13], a routing protocol was introduced by S.B. Alla et al. which implements a static or fixed cluster along with dynamic cluster head selection during every round. CCPR eliminates the need for cluster formation again and again by partitioning the sensing area into equal subarea's or cell in the beginning of network operation for effective management of information sensed by nodes. Limitation of CCPR lies in the cluster head selection and placement of relay nodes which is not optimal for random and sparse distribution of sensor nodes unlike CCPR. Apart from this, static clusters formed after network partitioning in CCPR are not balanced if we assume random and sparse deployment of nodes. CCPR does not consider node density during selection of cluster head and the distance from base station during placement of relay nodes and also during data transmission. It can be concluded that inappropriate placement of relay node and selection of sensor node, having less number of surrounding node as cluster head along with unbalanced clusters in CCPR, results in huge loss of connectivity and network lifetime during data transmission.

\section{SYSTEM MODEL'S USED}

\subsection{Network Model and Basic Assumptions}

We assume that set of " $n$ " heterogeneous sensors are deployed in an area. Each sensors node has an ID, a transmission or communication range as $\mathrm{R}_{\mathrm{C}}$, and a sensing radius as $\mathrm{R}_{\mathrm{S}}$. All heterogeneous sensor nodes are aware of their location coordinates and capable of reporting the particular location where the information is sensed. It has been proved that a single sink node responsible for collecting all information from the wireless sensor network is not an effective solution while dealing with large size wireless sensor network. As the network grows, the amount of data which be delivered to the sink or base station by nodes increases data traffic resulting in energy consumption bottlenecks. A better alternative solution [3] is based on making use of additional nodes, called relay node playing the role of a routers that helps in load balancing and keep length of multi-hop paths within a reasonable length as shown in Figure 3.1.1. In this proposed work selection and placement of relay node and cluster head are the key challenges for designing energy and density aware networks. Sensor nodes deployed in monitoring are need to be divided further into individual subsets that can send their traffic to a relay node via cluster head resulting in requirement of efficient clustering problem. The basic system model is depicted in Figure 3.1.1. Every node sends the sensed data to its respective allocated cluster head. Cluster head after receiving data, performs data aggregation and data fusion followed by data transmission to it's respective relay node that will forward data to the base station. We have considered a network scenario where relay nodes are connected to base station (BS) and thus acting as wireless routers and are responsible for forwarding all traffic generated by following multi hop communication path toward a base station [3].
Following are the assumptions about network setup:

- Network is located in $\mathrm{N}^{*} \mathrm{~N}$ square area and base station (BS) is located outside the monitoring area.

- Random deployment along with uneven or sparse distribution of heterogeneous wireless sensor nodes is done after which battery can't be recharged again.

- Nodes have heterogeneity in terms of energy i.e. different energy level. Some nodes are having more energy than other nodes.

- Base station and all other wireless sensor nodes are stationary after deployment and aware of their location.

- Nodes sense the region regularly and most of the time they have data which is to be delivered to base station.

- All sensor nodes can vary their transmitting power as per requirement because sensor nodes are equipped with module having power control capabilities.

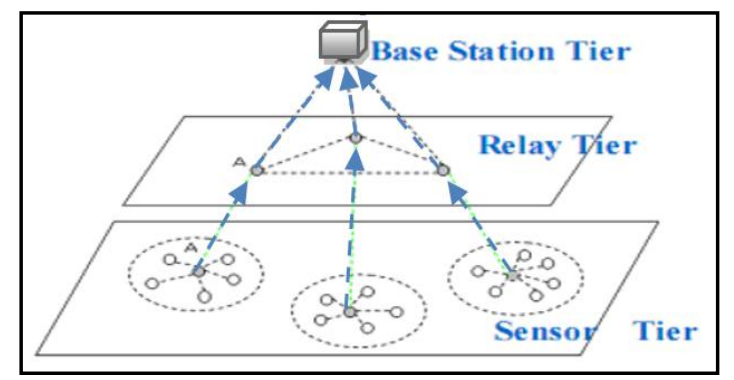

Figure 3.1.1: Proposed EDAC Protocol Network Model

\subsection{Coverage Model}

We assume that the targeting area is two dimensional plane and of size, $\mathrm{N} * \mathrm{~N}$ square sensing field. The " $\mathrm{n}$ ", number of the heterogeneous wireless sensor nodes with the same parameter value are put on this $2 \mathrm{D}$ plane, the coordinates of every sensor nodes are known in advance, and the sensing radius is $r_{i}$, transmission or communication radius is $R_{i}$ where $R_{i}=$ $\left(2 * r_{i}\right)$.We refer to these set of heterogeneous wireless sensor nodes which has been deployed in the monitoring area as $\mathrm{S}=\left\{\mathrm{S}_{1}, \mathrm{~S}_{2}, \mathrm{~S}_{3} \ldots \ldots . \mathrm{S}_{\mathrm{n}}\right\}$ and sensor node is represented as $\mathrm{Si}=\left\{\mathrm{x}_{\mathrm{i}}, \mathrm{y}_{\mathrm{i}}, \mathrm{r}_{\mathrm{i}}\right\}$. The monitoring or target area is equally digitized [14] into $\mathrm{N}^{*} \mathrm{~N}$ pixels array and size of each pixel is equal to 1 unit .The coverage model of each sensor $\mathrm{S}_{\mathrm{i}}$ can be considered as circle with center having its coordinates as $\left(\mathrm{x}_{\mathrm{i}}, \mathrm{y}_{\mathrm{i}}\right)$ and radius as " $r_{i}$ ". A random variable " $\mathrm{C}_{i}$ " will describe the event that a pixel $(\mathrm{x}, \mathrm{y})$ is covered by the senor $\mathrm{S}_{\mathrm{i}}$. The probability of an event $\mathrm{C}_{\mathrm{i}}$ is denoted as $\mathrm{P}\left(\mathrm{C}_{\mathrm{i}}\right)$, is equal to the coverage probability $\mathrm{P}_{\text {cov }}\left(\mathrm{x}, \mathrm{y}, \mathrm{S}_{\mathrm{i}}\right)$.This will generate binary valued function as shown below.

$$
\mathbf{P}\left(\mathbf{C}_{\mathbf{i}}\right)=\mathbf{P}_{\mathbf{c o v}}\left(\mathbf{x}, \mathbf{y}, \mathbf{S}_{\mathrm{i}}\right)\left\{\begin{array}{ll}
1 & \text { if }\left(\mathrm{x}-\mathrm{x}_{\mathrm{i}}\right)^{2}-\left(\mathrm{y}-\mathrm{y}_{\mathrm{i}}\right)^{2}<\mathrm{r}_{\mathrm{i}}^{2} \\
0 & \text { otherwise }
\end{array}\right\}
$$

\subsection{Radio Energy Dissipation Model}

First order radio model [5] has been used for the radio hardware energy dissipation in order to achieve acceptable SNR. The transmitter dissipates energy to run the radio electronics, power amplifier and the receiver dissipates energy to run the radio electronics is shown in Figure 3.3.1 [5]. The 
formula for sending and receiving a message are as given and explained in detail.

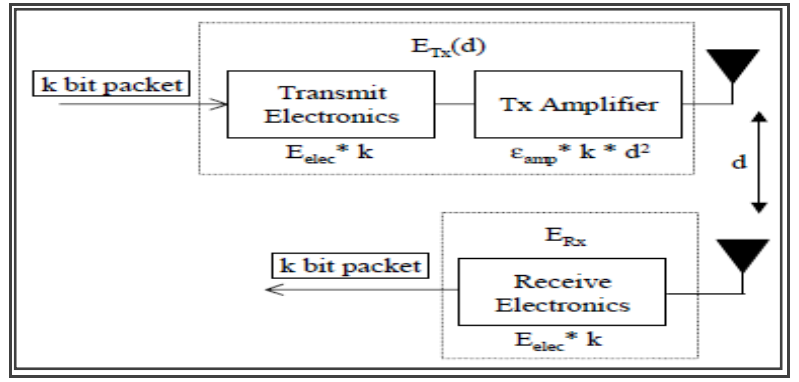

Figure 3.3.1: Radio Energy Dissipation Model [5]

Energy required to transmit and receive a L-bit packet is given as follows by $\mathrm{E}_{\mathrm{TX}}=\mathrm{L} *\left(\mathrm{E}_{\mathrm{elec}}+\mathrm{E}_{\mathrm{fs}}{ }^{*} \mathrm{~d}^{\mathrm{p}}\right)$ and $\mathrm{E}_{\mathrm{Rx}}=\mathrm{E}_{\text {elec }} * \mathrm{~L}$ where $\mathrm{E}_{\mathrm{Tx}}$ and $\mathrm{E}_{\mathrm{Rx}}$ are the energy consumption for transmitting data and receiving data respectively. "d" represent distance between the transmitter node and the receiver node and $p$ is the path loss exponent. If $\left(\mathrm{d}<\mathrm{d}_{0}\right)$ where, $\mathrm{d}_{\mathrm{o}}=\left(\left(\mathrm{E}_{\mathrm{fs}} / \mathrm{E}_{\mathrm{amp}}\right)^{\wedge} .5\right)$ then free space channel i.e. $(\mathrm{p}=2)$ is used otherwise multipath fading channel $(\mathrm{p}=4)$ model will be used in this proposed scheme . The parameters $\mathrm{E}_{\mathrm{elec}}$ and $\mathrm{E}_{\mathrm{fs}}$ are the parameters of the transmission and reception circuitry. For the sensor nodes serving as cluster head, apart from energy consumed in transmitting and receiving data, some extra energy is also consumed to complete the tasks of data aggregating and data compression. This extra energy, denoted as $\mathrm{E}_{\mathrm{CH}}$ and is calculated as $\mathrm{E}_{\mathrm{CH}}=\mathrm{E}_{\mathrm{Rx}}+\mathrm{L} * \mathrm{E}_{\mathrm{DA}}+\mu * \mathrm{E}_{\mathrm{Tx}}$ where $\mathrm{E}_{\mathrm{DA}}$ is the energy consumed per bit during data aggregation and $\mu$ is the compression coefficient.

\section{PROPOSED EDAC ALGORITHM}

Communication energy consumed while transmitting data through distance " $\mathrm{d}$ " is directly proportional to $\mathrm{d}^{\mathrm{p}}$ where " $\mathrm{d}$ " is the distance between transmitter and receiver .According to first order radio energy dissipation model, value of $\mathrm{p}$ can be either equal to two in case of frees space channel model or it can be four for multipath fading channel. Static clustering along with dynamic cluster head selection [20], [21] during every round in EDAC also saves computation overhead involved in cluster formation again and again. Thus it can be concluded that by implementing static clusters and multi-hop communication between sensor nodes and cluster head using relay nodes would definitely result in load balancing and better connectivity in the entire heterogeneous wireless sensor network.

In EDAC, energy heterogeneity up to three level has been implemented for better network lifetime and connectivity considering optimal selection of cluster head $(\mathrm{CH})$ and relay node (RN). Placement of cluster head and relay node has also been optimized further for gaining better efficiency in terms of network lifetime and connectivity by considering node density of nodes, coverage model and distance with respect to base station and elected relay node for each successive round. In the following sub-sections each phases of EDAC is discussed in brief.

\subsection{Setup Phase}

In setup phase fixed static clusters or cells on the basis of division of $\mathrm{N}^{*} \mathrm{~N}$ square sensing field are formed only once at the beginning of network operation. To accomplish this task base station divide the monitoring area into $\mathrm{k}$ equal sub area's or cells where, $\mathrm{k}$ is defined as $(\mathrm{N} / \mathrm{u})$ and $\mathrm{u}$ is calculated as $\mathrm{u}=(\mathrm{N} / 4),(\mathrm{N} / 6),(\mathrm{N} / 8), \ldots$ as per sensing requirement which will directly effect the number of cluster heads formed. For instance, by setting the value of $\mathrm{u}$ as 25 , exactly $\mathrm{k}=4$ static clusters will be formed. Sensor nodes on the basis of localization technique and few GPS enabled sensor's ,will determine their respective location with respect to sub area or cell and inform base station which will maintain key value pair in the form of (node_id,cell_id). By broadcasting k-1 different groups of messages which contains unique key value pair having node_id and cell_id as k-1,k-2 or k-3. Each sensor will fetch it's respective cell_id on the basis of it's own node_id. At last all nodes which have not joined any cluster or cell yet will set there cell_id as k and inform base station which will again verify the key value pair in it's database. Center of each cell is denoted by "RP" for use in next phase.

\subsubsection{Coverage-Aware Cost Metric}

The sensing area of each node can be considered as a circle surrounding the node having radius, $\mathrm{R}_{\mathrm{S}}$. A set of POI's i.e. point of interest in sensing field that will be monitored by sensor node are represented by $\mathrm{P}$ and defined as $\mathrm{P}=\{\mathrm{P}, \mathrm{j}=$ $1,2,3 \ldots \ldots . . . \mathrm{m}\}$.If the distance between POI $\left(\mathrm{P}_{\mathrm{j}}\right)$ and a sensor node $S_{i}$ is either less than or equal to $R_{S}$, then coverage set [12] of the node $S_{i}$ is given by $C\left(S_{i}\right)=\left\{p_{j} \mid d\left(S_{i}, P_{j}\right)<=R_{S}\right\}$ where $d\left(S_{i}, P_{j}\right)$ denotes Euclidean distance between the node $S_{i}$ and POI $\mathrm{p}_{\mathrm{j}}$. Generally multiple sensor nodes in the WSN may cover the same POI's. This is known as coverage redundancy. According to the definition mentioned above, the subset of POls that are simultaneously covered or sensed by multiple wireless sensor nodes can be calculated as:

$\mathrm{O}\left(\mathrm{S}_{\mathrm{i}}\right)=\left(\mathrm{C}\left(\mathrm{S}_{\mathrm{i}}\right) \cap\left(\mathrm{U}_{\mathrm{j}=1}^{\mathrm{n}} \mathrm{C}(\mathrm{Sj})\right)\right.$ where $\mathrm{O}\left(\mathrm{S}_{\mathrm{i}}\right)$ denotes the intersection of the sets of POI's covered by node $S_{i}$ and other close sensor nodes in the vicinity. If $\mathrm{C}\left(\mathrm{S}_{\mathrm{j}}\right)=\mathrm{O}\left(\mathrm{S}_{\mathrm{i}}\right)$, then sensor node $S_{\mathrm{i}}$ is identified as redundant node.

\subsection{Responsible Node Selection Phase}

Setup phase is followed by responsible node selection phase in which optimal selection and placement of responsible nodes like cluster head and relay node is done to improve connectivity between nodes. Before the start of each round base station which is very high computing device will compute parameters like energy ratio i.e. $\left(\mathrm{Q}_{\mathrm{i}}\right)$, node density i.e. $\mathrm{ND}\left(\mathrm{S}_{\mathrm{i}}\right)$, overlapping ratio i.e. $\left(\mathrm{O}\left(\mathrm{S}_{\mathrm{i}}\right) / \mathrm{C}\left(\mathrm{S}_{\mathrm{i}}\right)\right)$ of nodes and distance of node from base station i.e. (BS) and center of its respective cell i.e. (RP). After this Cluster head $(\mathrm{CH})$ and relay node ( $\mathrm{RN}$ ) are selected on the basis of various parameters which are known to base station in advance.

\subsubsection{Selection of Relay Node}

Using the formula given below we will find the node having the highest value of Cost Factor, $\left(\mathrm{S}_{\mathrm{i}}\right)$ to determine most eligible sensor node fit for becoming relay node $\left(\mathrm{R}_{\mathrm{N}}\right)$ during particular round. Constant such as w1 and w2 are the weighting coefficients which helps to adjust the relative importance of the energy ratio factor and the distance to the center of cluster (cell) or base station factor, respectively.

$$
\text { Cost Factor }\left(\mathrm{S}_{\mathrm{i}}\right)=\left(\mathrm{Q}_{\mathrm{i}}\right)^{\mathrm{w} 1}{ }_{*}\left(1 / \mathrm{d}\left(\mathrm{S}_{\mathrm{i}}, \mathrm{RP}\right)\right)^{\mathrm{w} 2}{ }_{*}\left(1 / \mathrm{d}\left(\mathrm{S}_{\mathrm{i}}, \mathrm{BS}\right)\right)^{\mathrm{w} 2}
$$

\subsubsection{Selection of Cluster Head}

Using the formula given below we will find the node having highest value of Cost Factor, $\left(\mathrm{S}_{\mathrm{i}}\right)$ to determine most eligible sensor node fit for becoming cluster head $(\mathrm{CH})$ during particular round. Constant such as $\beta 1, \beta 2$ and $\beta 3$ are the weighting coefficients which helps to adjust the relative importance of the energy ratio factor, the coverage density 
cost factor and the distance to the location of elected relay node $\left(\mathrm{R}_{\mathrm{N}}\right)$ factor, respectively.

Cost Factor $\left(\mathrm{S}_{\mathrm{i}}\right)=\left(\mathrm{Q}_{\mathrm{i}}\right)^{\beta 1}{ }_{*}\left(\mathrm{O}\left(\mathrm{S}_{\mathrm{i}}\right) / \mathrm{C}\left(\mathrm{S}_{\mathrm{i}}\right)\right)^{\beta 2}{ }_{*}\left(1 / \mathrm{d}\left(\mathrm{S}_{\mathrm{i}}, \mathrm{R}_{\mathrm{N}}\right)\right)^{\beta 3}{ }_{*} \mathrm{ND}\left(\mathrm{S}_{\mathrm{i}}\right)$

\subsection{Steady State Phase}

During this phase of EDAC, each non cluster head sensor nodes will join elected cluster head to form respective cluster located in particular cell inside sensing field. Each sensor node will send "JOIN" messages along with there node_id to inform cluster head of respective cell or cluster about there membership. Cluster head $(\mathrm{CH})$ will reply them back with same JOIN message by appending details like TDMA code_id and node_id in message header thus replying them with acknowledgement of approval of there membership. TDMA will ensure that sensor nodes send their data to $\mathrm{CH}$ during pre allocated time slot only in order to avoid collision of data.

Once cluster are defined, the data transmission phase begins where the each sensor nodes according to there respective TDMA schedule will send its collected data to cluster head. The cluster head $(\mathrm{CH})$ after performing data aggregation will route the data packets over the well defined multi hop paths to base station or sink by making use of relay node. Cluster head will take its decision of sending its data directly to base station or to relay node on the basis of two conditions given below. Here $\mathrm{D}_{\mathrm{CH}} \mathrm{To}_{-} \mathrm{D}_{\mathrm{BS}}$ represents distance between cluster head and base station, $\mathrm{D}_{\mathrm{CH} \_}$To_ $\mathrm{D}_{\mathrm{RN}}$ represents distance between cluster head and relay node and $\mathrm{D}_{\mathrm{RN}-} \mathrm{To}_{-} \mathrm{D}_{\mathrm{BS}}$ represents distance between relay node and base station.

1) If $\mathrm{D}_{\mathrm{CH} \_}$To_D $\mathrm{D}_{\mathrm{BS}}=>\left(\mathrm{D}_{\mathrm{CH}-\mathrm{To} \_} \mathrm{D}_{\mathrm{RN}}+\mathrm{D}_{\mathrm{RN} \_} \mathrm{To}_{-} \mathrm{D}_{\mathrm{BS}}\right)$

Then collected data from cluster head will be transmitted to the Base Station via relay node following multi hop communication path to save energy.

2) If $\mathrm{D}_{\mathrm{CH} \_} \mathrm{To} \_\mathrm{D}_{\mathrm{BS}}<=\left(\mathrm{D}_{\mathrm{CH} \_} \mathrm{To} \_\mathrm{D}_{\mathrm{RN}}+\mathrm{D}_{\mathrm{RN} \_}\right.$To_D $\left.\mathrm{D}_{\mathrm{BS}}\right)$

Then cluster head will send collected data directly to the base station without using relay node to save energy.

\section{SIMULATION}

We conduct simulation using most popular and efficient simulation tool, MATLAB R2012a [15] by considering scenario used in real life applications like wireless air pollution monitoring system or radioactive emission detection system which requires division of sensing field into equal subarea's [18] for better management of data flow. We have used same simulation parameters used in CCPR [13] to make fair comparison of results obtained. Network with 100, two level heterogeneous wireless sensor nodes are deployed in random and sparse i.e. in uneven manner as opposed to CCPR which considers even or well distributed deployment of sensor nodes over sensing field of size $50 \times 50 \mathrm{~m}^{2}$ as shown in Figure 5.2.1 The sensing range, $R_{s}$ is set at $7.5 \mathrm{~m}$ and communication range, $R_{c}$ is set at $15 \mathrm{~m}$ which can be adjusted as per requirement of data transmission. In addition, this area consists of 2,500 POI's, distributed in grid like manner. In each round alive nodes need to report the sensed data to the BS and the BS is located at the coordinate $(25,75)$. The initial energy of normal node is assumed to be 1 joule in the initial time. For the transmission between nodes, $\mathrm{p}$ is assumed to be 2 whereas $\mathrm{p}$ is assumed to be 2.5 for the transmission from a relay nodes to the BS.The compression coefficient $\mu$ is set to 0.05 which is much suitable for the real-world tasks [7]. After Simulation using quantitative analysis, optimum value of network lifetime having $100 \%$ sensing coverage ratio was obtained when value of constant, $w 1=.8$ and $w 2=.2$. Similarly optimal values for constant, $\beta 1, \beta 2$ and $\beta 3$ were found to be as $1, .8$ and .2 respectively for $100 \%$ sensing coverage ratio. Furthermore, we ignore the effect caused by signal collision and interference in the wireless channel. The various parameters of the radio model are summarized in Table 5.1.1.

\subsection{Performance Metrics:}

- Number of alive (normal nodes, advance nodes , super nodes and super advance node) versus network lifetime.

- Stability Period: It is the time interval between start of network and the death of first working or alive node [7].

- Network Lifetime: It is the time interval between start of network and the death of last working or alive node.

Table 5.1.1: Simulation Parameters

\begin{tabular}{|c|c|c|}
\hline Notation & Description & Value \\
\hline $\mathbf{N} \times \mathbf{N}$ & Sensing Area & $50 \times 50$ \\
\hline $\mathbf{n}$ & Number of the sensor nodes & 100 \\
\hline $\begin{array}{l}\text { Sink } \\
\text { Location }\end{array}$ & Base Station or Sink location & $(25,75)$ \\
\hline $\mathbf{E}_{\mathbf{o}}$ & Initial energy & $1 \mathrm{~J}$ \\
\hline $\mathbf{E}_{\text {elec }}$ & Electronics energy & $50 \mathrm{~nJ} / \mathrm{bit} / \mathrm{message}$ \\
\hline $\mathbf{E}_{\mathrm{DA}}$ & Energy of data aggregation & $5 \mathrm{~nJ} / \mathrm{bit} / \mathrm{m}^{2}$ \\
\hline $\mathbf{E}_{\mathrm{fs}}$ & $\begin{array}{l}\text { Amplified energy for transmitting } \\
\text { in free space model }\end{array}$ & $.1 \mathrm{~nJ} / \mathrm{bit} / \mathrm{m}^{2}$ \\
\hline $\mathbf{E}_{\text {amp }}$ & $\begin{array}{l}\text { Amplified energy for transmitting } \\
\text { in multipath model }\end{array}$ & $0.0013 \mathrm{pJ} / \mathrm{bit} / \mathrm{m}^{4}$ \\
\hline $\mathbf{L}$ & Data packet size & 200bytes \\
\hline $\mathbf{u}$ & Constant for calculating subarea & 25 \\
\hline $\mathbf{k}$ & Number of Relay Nodes (RN) & 4 \\
\hline $\mathbf{P}$ & Path loss exponent & 2 or 2.5 \\
\hline$\mu$ & Compression coefficient & .05 \\
\hline
\end{tabular}

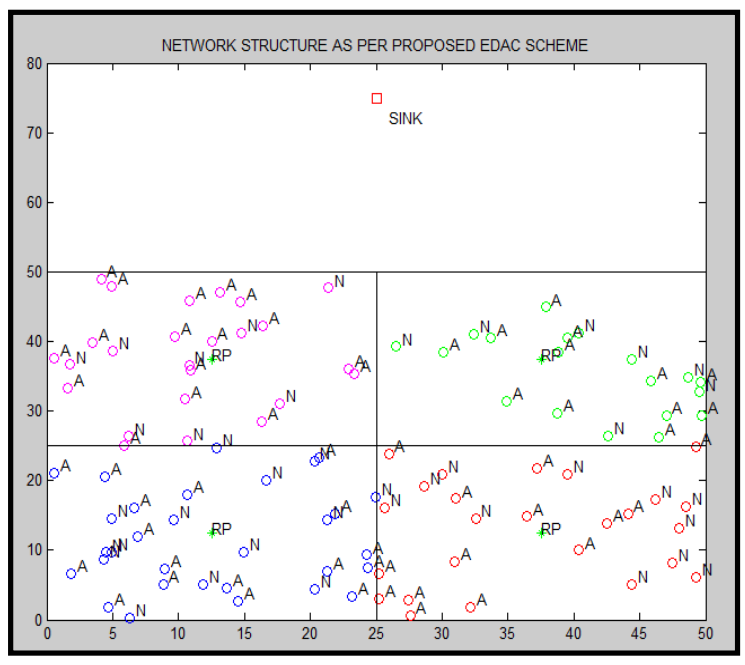

Figure 5.2.1: Network partition in EDAC where normal nodes are denoted by ' $N$ ' and advance node by ' $A$ ' 


\subsection{Results:}

During simulation we considered 2 level heterogeneity and deployed $60 \%$ advanced nodes represented by ' $A$ ', having one times more energy than normal nodes which are represented by ' $\mathrm{N}$ ' as shown in Figure 5.2.1. Using rand() and random() function of Matlab ,deployment of nodes is done in random and sparse i.e. in uneven manner. We have compared our proposed scheme, EDAC with existing protocol, CCPR [13] which is too extended up to two energy level, based upon same approach. Random and uneven deployment of wireless sensor nodes has been considered which is applicable in most real life applications like air pollution monitoring system [18], radioactive pollution monitoring system, sensor based volcano monitoring system etc.

In Figure 5.2.2, a performance of EDAC and CCPR is illustrated using graph obtained after performing simulation in Matlab 2012a. Graph is showing relation between alive nodes, represented along $\mathrm{x}$ axis versus network lifetime(), represented along y axis. Network lifetime (denoted as number of rounds) of EDAC is 4308 round which is much better than CCPR having network lifetime of just 1860.The first node dies at $1202^{\text {th }}$ round in EDAC as compared to CCPR where first node dies at $667^{\text {th }}$ round, indicating increase in stability period of network. The last node dies at $4308^{\text {th }}$ round in EDAC which is better in comparisons to CCPR where last node die at just $1860^{\text {th }}$ round. In case of EDAC $50 \%$ of nodes dies at $1758^{\text {th }}$ round and in CCPR 50\% nodes dies at $1070^{\text {th }}$ round clearly indicating better connectivity and coverage obtained in EDAC. Thus it can be concluded that EDAC is $80.21 \%$ more efficient in terms of $100 \%$ coverage ratio and connectivity in comparison to CCPR. EDAC is $64.3 \%$ more better in terms of network lifetime as compared to CCPR considering 50\% remaining alive node under same simulation environment and parameter values. Thus it can be further concluded from Figure 5.2.3 that EDAC is better performer than CCPR considering parameter like stability period, network lifetime, coverage ratio ( for $100 \% \& 50 \%$ node dead) and connectivity.

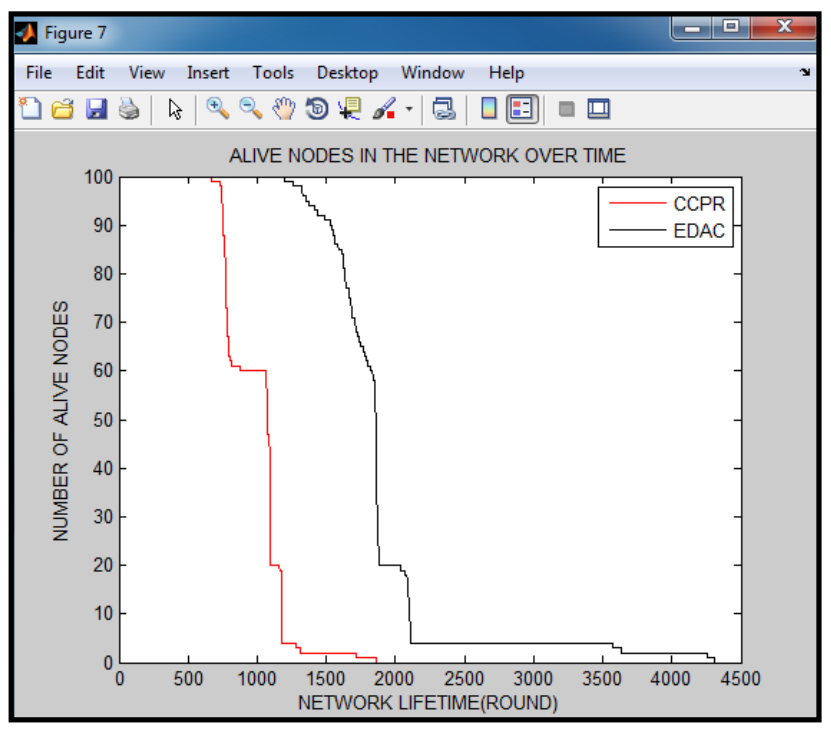

Figure 5.2.2: Number of alive sensor nodes versus network lifetime (rounds) under 2 Level energy heterogeneity

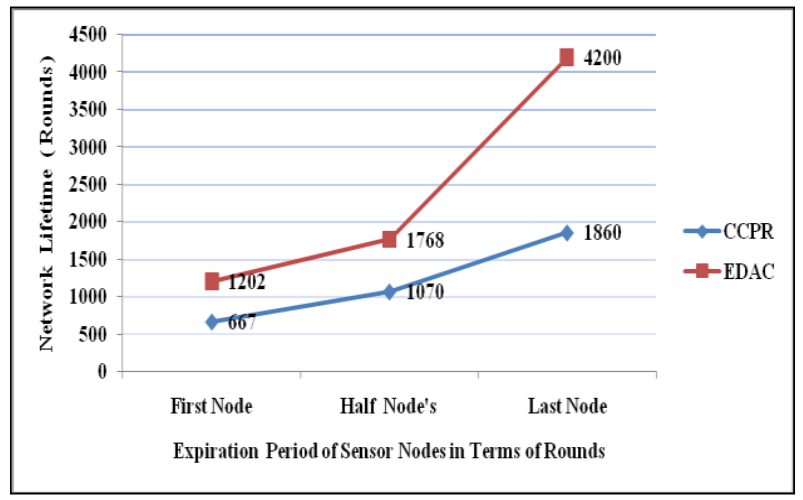

Figure 5.2.3: Coverage and Lifetime performance of CCPR and EDAC under 2 Level energy heterogeneity

\section{CONCLUSION \& FUTURE SCOPE 6.1 CONCLUSION}

Heterogeneous wireless sensor network consists of more than one type of sensor nodes which differs from one another either in terms of initial energy, computing power or performance. Today most of the deployment of sensor networks is done in random manner because of remote or hostile environment. Therefore objective to increase network lifetime and connectivity in wireless sensor network must be fulfilled while developing routing protocol for WSN. In this paper ,EDAC adds heterogeneity in terms of energy level to the sensor network by introducing advance nodes having more energy than normal nodes. Simulation done on MATLAB 2012a shows that proposed EDAC has better performance when compared to CCPR in terms of parameter like stability period, $100 \%$ coverage ratio, connectivity and network lifetime.

\subsection{FUTURE SCOPE}

This work can be extended and tested for higher Level of energy heterogeneity for studying the effects of higher energy heterogeneity in the network lifetime. In addition to this, static cluster formation in EDAC should be further optimized to make them balanced in terms of number of nodes as in case of deterministic deployment which have well spread and evenly distributed wireless sensor nodes. Apart from this introduction of mobile sensor nodes can be introduced to reduce coverage holes and ensure smooth connectivity which is not available during random deployment of sensor nodes where nodes are not evenly distributed. All these issues are still left to be implemented as a future work.

\section{ACKNOWLEDGEMENTS}

I express my deepest gratitude to my supervisor, Neha Garg for her motivational support for writing this paper. She gave her regular feedbacks and innovative ideas from time to time. I also express my humble gratitude to Dr. Santosh Kumar and Mr. Devesh Pratap Singh for their constructive feedbacks and comments which helped me to further improve my work. I also wish to acknowledge Graphic Era University, Dehradun, Uttarakhand, India for providing all the necessary facilities. At last I would like to thanks the anonymous reviewers for their helpful comments and suggestions. 


\section{REFERENCES}

[1] Ian F. Akyildiz et al.,"A Survey on Sensor Networks", IEEE Communications Magazine August ,2009.

[2] J. N. Al-Karaki and A. E. Kamal, "Routing Techniques in Wireless Sensor Networks: A Survey", IEEE Wireless Communication Vol.11, No.6, Dec.2004.

[3] Capone, Antonio, Matteo Cesana, Danilo De Donno, and Ilario Filippini. "Deploying multiple interconnected gateways in heterogeneous wireless sensor networks: An optimization approach." Computer Communications 33, no. 10 (2010): 1151-1161.

[4] Ian F Akyildiz, Tommaso Melodia, and Kaushik R. Chowdhury,"Wireless multimedia sensor networks: Applications and testbeds ", Proceedings of the IEEE, vol 96, pp. 1588-1605,2008

[5] W. R. Heinzelman A. Chandrakasan, and H. Balkrishnan, "An Application-Specific Protocol Architecture for Wireless Microsensor Networks", IEEE Trans. Wireless Communication, Vol. 1, No. 4, pp. 660670,October 2002.

[6] Wendi Rabiner Heinzelman, Anantha Chandrakasan, and Hari Balakrishnan,"Energy-efficient communication protocol for wireless microsensor networks", 2000,Proceedings of the 33rd annual Hawaii international conference on In System sciences, IEEE, 2000 .

[7] Georgios S. et al. ,"SEP: A stable election protocol for clustered heterogeneous wireless sensor networks", Boston University Computer Science Department , 2004.

[8] Said Benkirane et al. "Distance-based Stable Election Protocol (DB-SEP) for Heterogeneous Wireless Sensor Network", IJCA, vol.58, pp. 9-15, 2012.

[9] Li Qing, Qingxin Zhu and Mingwen Wang,"Design of a distributed energy-efficient clustering algorithm for heterogeneous wireless sensor networks" ,Computer communications, Elsevier, vol. 29,pp. 2230-2237, 2006.

[10] Mark Yarvis et al. ,"Exploiting heterogeneity in sensor networks",24th Annual Joint Conference of the IEEE Computer and Communications Societies, IEEE. Vol. 2, 2005.

[11] Stanislava Soro and Wendi B. Heinzelman, "Cluster head election techniques for coverage preservation in wireless sensor networks", Ad Hoc Networks, Elsevier,vol.7, pp.955-972,2009.
[12] Joe Air Jiang et al.,"A QoS-guaranteed coverage precedence routing algorithm for wireless sensor networks.", Sensors vol. 11,pp.3418-3438,2011.

[13] Said Ben Alla and Abdellah Ezzati ,"Coverage and connectivity preserving routing protocol for heterogeneous wireless sensor networks", In Next Generation Networks and Services (NGNS), 2012, pp. 141-148,IEEE, December, 2012.

[14] Jie Jia, Xueli Wu, Jian Chen, and Xingwei Wang, "Exploiting sensor redistribution for eliminating the energy hole problem in mobile sensor networks", EURASIP, Journal on Wireless Communications and Networking, Springer vol.1,2012.

[15] Matlab Simulator, URL , https://www.mathworks.com/ products/ matlab /

[16] Prachi Uplap et al.,"Review of Heterogeneous/ Homogeneous Wireless Sensor Networks and Intrusion Detection System Techniques ", ACEEE, Association of Computer Electronics and Electrical Engineers, 2014.

[17] Basit Manzoor et al.,"Q-LEACH: A new routing protocol for WSNs.", Procedia Computer Science, Elsevier, vol. 19, pp. 926-931, 2013

[18] Kavi K. Khedo, Rajiv Perseedoss, and Avinash Mungur, "A Wireless Sensor Network Air Pollution Monitoring System", International Journal of Wireless \& Mobile Networks vol. 2, no. 2 ,pp. 31-45,2010

[19] Singh, Shio Kumar, M. P. Singh, and D. K. Singh. "Energy-efficient Homogeneous Clustering Algorithm for Wireless Sensor Network", International Journal of Wireless \& Mobile Networks , pp. 49-61, vol 2.3, 2010.

[20] A. Ahmad et al, "Density controlled divide-and-rule scheme for energy efficient routing in Wireless Sensor Networks", In $26^{\text {th }}$ Annual IEEE Canadian Conference of Electrical and Computer Engineering, IEEE ,2013.

[21] Abrar Haider, Nadeem Javaid, N. Amjad, A. A. Awan, Ajmal Khan, and Noel Khan, "REECH-ME: Regional energy efficient cluster heads based on maximum energy routing protocol for WSNs." In $8^{\text {th }}$ International Conference on Broadband and Wireless Computing, Communication and Applications (BWCCA), pp. 88-92, IEEE, 2013

[22] Ajit Singh Negi, Neha Garg, and Akhand Pratap Singh, "Role of Clustering in Achieving Energy Efficient Coverage in Wireless Sensor Network: A Short Review", IRJET, vol 2, issue 2, May , 2015.

$[23]$ 